\title{
Epipolar Visual Servoing for Multi-Robot Distributed Consensus
}

\author{
Eduardo Montijano, Johan Thunberg, Xiaoming Hu, Carlos Sagues
}

\begin{abstract}
In this paper we give a distributed solution to the problem of making a team of non-holonomic robots reach consensus about their orientations using monocular cameras. We consider a scheme where the motions of the robots is decided using nearest neighbor rules. Each robot is equipped with a camera and can only exchange visual information with a subset of the other robots. The main contribution of this paper is a new controller that uses the epipoles computed from the images provided by neighboring robots, eventually reaching consensus in their orientations without the necessity of directly observe each other. In addition, the controller only requires a partial knowledge of the calibration of the cameras in order to achieve the desired configuration. We also demonstrate that the controller is robust to changes in the topology of the network and we use this robustness to propose strategies to reduce the computational load of the robots. Finally, we test our controller in simulations using a virtual environment and with real robots moving in indoors and outdoors scenarios.
\end{abstract}

\section{INTRODUCTION}

The idea of multiple robots working cooperatively to achieve a common goal is of high interest in many tasks such as exploration, surveillance or transportation. Multi-robot systems can perform these tasks with more robustness or in less time than one robot working alone. On the other hand, in order to carry on with these tasks, the robots need to be able to move coordinately. A generalized problem in the context of coordinated motion is the problem of reaching a consensus by all the robots. The consensus problem we focus on is to make a team of robots move all together in a common direction [1], [2]. Reaching a consensus in the orientations of the robots is of interest in other robotic applications, for example it can simplify data association or map alignment problems that appear in cooperative exploration situations, reducing them to linear translation problems [3], [4].

Some approaches achieve consensus using leader-follower schemes [5]-[8], where each follower-robot designs its control input using the information provided by its leader in the team. Nearest-neighbor rules, where the robots design their control input using all the available information provided by direct

E. Montijano is with Centro Universitario de la Defensa and Instituto de Investigación en Ingeniería de Aragón (I3A), Zaragoza, Spain.

J. Thunberg and X. Hu are with Department of Math, Division of Optimization and Systems Theory, Royal Institute of Technology (KTH), Sweden.

C. Sagues is with Departamento de Informática e Ingeniería de Sistemas - Instituto de Investigación en Ingeniería de Aragón (I3A), Universidad de Zaragoza, Spain.

This work was supported by the projects DPI2009-08126 and DPI201232100 and partly supported by Swedish Research Council and Swedish Foundation for Strategic Research. The authors would like to thank L. Riazuelo and D. Tardioli for their help with the real experiments. neighbors in the communication graph are more appealing [9][15] than the previous ones. In these kind of approaches all the robots play the same role in the formation, conferring the system a natural robustness against changes in the topology and individual failures. A key aspect left aside in most of the existing works in this topic, e.g., [9]-[13], is how the robots estimate the position of their neighbors to control their motion.

Vision sensors can play a fundamental role in this part of the process due to the large amount of information that images contain. There are numerous works dealing with the problem of controlling a single robot using a vision system, e.g., [16]-[18]. Cameras have also been used in different multirobot scenarios such as pursuit-evasion [19], organized rendezvous [20], or multi-robot deployment [21], to name a few examples. Additionally, all the research done in the field of computer vision during the past decades can be exploited in a multi-robot framework in order to achieve the desired goal.

Regarding the consensus problem, distributed solutions using omnidirectional cameras can be found in [22], [23] where the robots can see all their neighbors. If the robots are equipped with monocular cameras with limited field of view then the observation of all the neighbors may not always be possible. A leader-follower solution is adopted in [7], where each robot has only one neighbor and is required to directly observe it in order to reach the consensus. All these approaches also have the drawback of requiring perfect knowledge of the intrinsic parameters of the cameras in order to reach the consensus. The control law we present in this paper contributes to the state of the art by relaxing these assumptions, allowing the robots to reach the consensus without the need of observing each other, topology restrictions or an exact knowledge of all the intrinsic parameters of the camera.

Our solution assumes that each robot moves in the plane with non-holonomic motion constraints and is equipped with a monocular camera with a limited field of view. In the presented approach each robot computes its control input using the epipoles between its current image and the images of its neighbors in the communication graph. A first approach using the epipoles to control the motion of a robot appeared in [24]. Non-holonomic constraints were introduced in [25][27]. However, in all the previous approaches the goal is to control one robot and move it to a fixed position, specified by some target image. In our approach we consider multiple robots moving simultaneously, using the epipoles computed between the images of the different robots at the same time instant. Additionally, since the images used to steer the robots are acquired at the same instant, the approach is robust to dynamic scenes. 
The main contribution of this paper is a non-linear distributed controller that makes use of the epipoles computed from the images of the different robots to achieve the consensus. We demonstrate that the controller is robust to changes in the topology of the network and use this result to propose two distributed strategies that allow the robots to reduce the number of fundamental matrices they need to compute at each iteration. In this way the team is still able to reach consensus but the computational demands of each robot are bounded and equal for all of them. In addition, the control only requires a partial knowledge of the calibration of the cameras in order to achieve the desired configuration. We validate our results in simulations and in real scenarios with real robots equipped with different cameras. A preliminary version of the controller can be found in [28] whereas the results about the neighbor selection appeared in [29]. Here we contribute by analyzing the influence of different cameras by the robots and also with extensive simulations and experiments with real robots, demonstrating the performance of our approach.

The rest of the paper is organized as follows: A detailed description of the robotic network considered in the paper is given in section II. In section III we present the vision-based consensus controller using epipoles and show its convergence for fixed connected communication topologies. More realistic situations are studied in section IV, where we analyze time-varying topologies, different cameras and we propose strategies to reduce the computational demands of the robots. Simulations and experiments with real robots demonstrate the good performance of our approach in sections $\mathrm{V}$ and VI respectively. Finally, in section VII we present the conclusions of the work.

\section{DESCRIPTION OF THE ROBOTIC NETWORK}

In this section we introduce the robotic network that describes the system where our controller is used.

\section{A. Dynamics of the robots}

We consider a set $\mathcal{V}$ of $N$ homogeneous autonomous robots moving on the plane with non-holonomic motion constraints,

$$
\left[\begin{array}{c}
\dot{x}_{i} \\
\dot{z}_{i} \\
\dot{\theta}_{i}
\end{array}\right]=\left[\begin{array}{cc}
\sin \left(\theta_{i}\right) & 0 \\
\cos \left(\theta_{i}\right) & 0 \\
0 & 1
\end{array}\right]\left[\begin{array}{c}
v_{i} \\
w_{i}
\end{array}\right] \text {, }
$$

where $\left[x_{i}, z_{i}, \theta_{i}\right]^{T}$ is the state of robot $i$ (position and orientation) expressed in some world reference frame and $\left[v_{i}, w_{i}\right]^{T}$ are the linear and angular velocity of the robot, respectively.

Given two robots, $i$ and $j$, their relative positions in the plane can be defined by a distance, $r_{i j}$, a bearing angle, $\psi_{i j}$, and relative orientation, $\theta_{i j}$, see Fig. 1 (a). The goal of the consensus problem is to make all the robots achieve the same orientation, i.e., $\theta_{i j} \rightarrow 0, \forall i, j \in \mathcal{V}$, as $t \rightarrow \infty$. Since the linear velocity is not required to make the robots achieve the consensus in the orientation, along the paper we consider it constant for all the robots, $v_{i}=v \geq 0, \forall i$.

\section{B. Communications between robots}

Communications between robots are modeled with an undirected graph $\mathcal{G}=(\mathcal{V}, \mathcal{E})$. The nodes in the graph represent the robots, $\mathcal{V}=\{1, \ldots, N\}$ whereas an edge models direct communication between two robots. In this way, if robots $i$ and $j$ are able to exchange messages with each other, then $(i, j) \in \mathcal{E}$. Since communications are undirected $(i, j) \in$ $\mathcal{E} \Leftrightarrow(j, i) \in \mathcal{E}$. The set of neighbors of robot $i$, i.e., the robots that can directly communicate with $i$, is defined as $\mathcal{N}_{i}=\{j \in \mathcal{V} \mid(i, j) \in \mathcal{E}\}$.

\section{Camera model and output of the system}

In our setup all the robots are equipped with pinhole monocular cameras with limited field of view. We assume that all the robots have identical cameras onboard with calibration matrix equal to $\mathbf{K}=\operatorname{diag}(\alpha, \alpha, 1)$, where $\alpha>0$ is the focal length of the camera measured in pixels. This is equivalent to say that the camera has no skew and that the principal point coincides with the center of the image. While the principal point and the skew are supposed to be known by the robots, we also assume that the exact value of $\alpha$ is not available to them. In sections and IV and V we analyze different situations where these assumptions do not hold.

The limited field of view of the cameras implies that in many situations the robots will not have direct line of sight with each other. To overcome this limitation the robots can exchange their images and use structure from motion techniques to estimate their neighbors positions (see Fig. 1 b). Unfortunately, since we are assuming that the robots do not know the exact value of $\alpha$, they cannot recover their relative positions and orientations from their images. In other words, the robots do not have direct access to $r_{i j}, \psi_{i j}$ and $\theta_{i j}$. Therefore, we require a different measurement that can be computed without the need of the exact calibration and that gives some idea to the robots about their relative orientations so that they can make them tend to zero.

In this paper we use the epipoles of the images acquired by neighboring robots to estimate and correct this misalignment. Given a pair of images, acquired by robots $i$ and $j$, it is possible to estimate the fundamental matrix, $\mathbf{F}_{i j}$, that relates them, provided that there are at least 7 correspondences between them [30]. After that, the epipoles, $e_{i j}=\left[e_{i j x}, e_{i j y}\right]^{T}$ and $e_{j i}=\left[e_{j i x}, e_{j i y}\right]^{T}$, can be computed using methods from linear algebra as the intersection of the epipolar lines defined by $\mathbf{F}_{i j}$ and the matched features. Due to the planar motion, the $y$-coordinate of all the epipoles will be equal and constant for any pair of images. The $\mathrm{x}$-coordinate of the epipoles satisfies

$$
e_{i j x}=\alpha \tan \left(\psi_{i j}\right), e_{j i x}=\alpha \tan \left(\psi_{i j}-\theta_{i j}\right) .
$$

For simplicity purposes, in the following we use $e_{i j}$ and $e_{j i}$ to refer to only the $\mathrm{x}$-coordinate of the epipoles.

\section{DISTRIBUTED CONSENSUS CONTROLLER USING EPIPOLES}

In this section we propose a control law for each robot that uses the epipoles as measurements to achieve the consensus. For the theoretical analysis we assume that the epipoles 


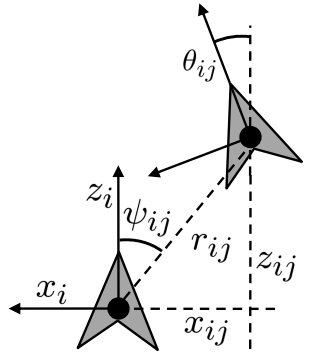

(a) (b)

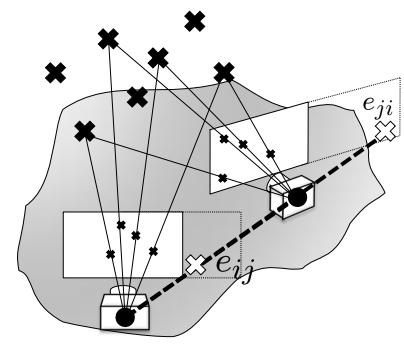

Fig. 1: (a) Coordinates of robot $j$ in the reference frame of robot $i$. (b) Observation of the neighbor robot with the epipoles. Both robots observe the same features in the scene and using structure from motion techniques they can compute the epipoles without the necessity of observing each other.

between neighbors in the communication graph are always computed without noise in their measurement. The influence of noise will be analyzed in the simulations of section $\mathrm{V}$.

Given a pair of neighbor robots, by eq. (2), a necessary condition for the attitude alignment is that their epipoles must be equal, $\theta_{i j}=0 \Rightarrow e_{i j}=e_{j i}$. However, note that $e_{i j}=e_{j i}$ does not necessarily imply consensus in the orientation because for $\theta_{i j}=\pi$ the two epipoles are also equal. This introduces the need to impose a constraint on the initial orientations of the robots. We require that initially $\left|\theta_{i j}\right|<\pi / 2, \forall i, j \in \mathcal{V}$, so that the controller is able to align the robots properly.

In order to reach the consensus each robot, $i$, computes the turning rate following this procedure: First it receives the visual information acquired for those robots that are neighbors in the communication graph $\mathcal{N}_{i}$. Then for each $j \in \mathcal{N}_{i}$ it computes the epipoles $\left(e_{i j}, e_{j i}\right)$. For each pair of epipoles, the robots compute a measurement of the relative misalignment by

$$
d_{i j}=\arctan \left(\frac{e_{i j}}{\beta}\right)-\arctan \left(\frac{e_{j i}}{\beta}\right) \in(-\pi, \pi],
$$

and

$$
w_{i j}=\left\{\begin{array}{ll}
d_{i j} & \text { if }\left|d_{i j}\right| \leq \frac{\pi}{2} \\
-\operatorname{sign}\left(d_{i j}\right)\left(\pi-\left|d_{i j}\right|\right) & \text { otherwise }
\end{array} .\right.
$$

with $0<\beta<\infty$ some fixed positive constant to choose.

Finally the control input is obtained summing the computed $w_{i j}$,

$$
w_{i}=K \sum_{j \in \mathcal{N}_{i}} w_{i j}
$$

with $K>0$ the controller gain.

There are several reasons to define the misalignment between pairs of robots in equations (3) and (4) rather than a simple difference between the epipoles, $e_{i j}-e_{j i}$. First of all, eq. (3) is a mapping $(-\infty, \infty) \rightarrow(-\pi, \pi)$, which clearly represents in a better way a relative orientation between two robots and leads to smaller control inputs. Secondly, equation (4) is used to select the closest path (clockwise or anticlockwise) that makes both epipoles be the same. In this way, the problem of epipoles at the infinity $\left(\psi_{i j} \simeq \pi / 2\right.$ and $\theta_{i j} \simeq 0$ ), which can fastly change their sign due to noise or small rotations is not an issue because the controller will always have values close to zero. If initially $\theta_{i j}<\pi / 2$, it also avoids the alignment of the epipoles in such a way that $\theta_{i j}=\pi$. To conclude, note that, if $\beta=\alpha$, then the setup is calibrated, $d_{i j}=\theta_{i j}$, and the relative orientation between the robots can be computed from the epipoles. However, we assume that this is not the case and $\beta \neq \alpha$.

In order to prove stability of the proposed controller, we will make use of the following lemma.

Lemma 3.1: The following properties hold:

1) $w_{i j}=-w_{j i}$.

2) $\sum_{i \in \mathcal{V}} w_{i}=0$

3) $\operatorname{sign}\left(e_{i j}\right)=\operatorname{sign}\left(e_{j i}\right) \Rightarrow\left|d_{i j}\right|<\pi / 2$.

Proof: First note that $d_{i j}=-d_{j i}$. Therefore, if $\left|d_{i j}\right| \leq$ $\pi / 2$, then $w_{i j}=-w_{j i}$. In eq. (4), when $\left|d_{i j}\right|>\pi / 2,(\pi-$ $\left.\left|d_{i j}\right|\right)$ has the same sign that $\left(\pi-\left|d_{j i}\right|\right)$ because $\left|d_{i j}\right| \leq \pi$. But $\operatorname{sign}\left(d_{i j}\right) \neq \operatorname{sign}\left(d_{j i}\right)$, which implies that $w_{i j}=-w_{j i}$ and 1) is proved.

The proof of 2) is done by decomposing the sum of $w_{i}$,

$$
\sum_{i \in \mathcal{V}} w_{i}=K \sum_{(i, j) \in \mathcal{E}} w_{i j} .
$$

Taking into account that the communication graph is undirected and $w_{i j}=-w_{j i}$, then the sum is equal to zero.

To prove 3) let us consider that both epipoles have the same sign, without loss of generality, positive. The arc tangents have values in the interval $[0, \pi / 2)$ and therefore, the difference in eq. (3) belongs to the interval $(-\pi / 2, \pi / 2)$.

Theorem 3.2: Consider a robotic network with the robots initially oriented in such a way that $\left|\theta_{i j}\right| \leq \theta_{M}<\pi / 2, \forall i, j \in$ $\mathcal{V}$. If the robots use the control law (5) with $\beta$ satisfying

$$
\alpha \tan \left(\frac{\theta_{M}}{2}\right)<\beta<\frac{\alpha}{\tan \left(\frac{\theta_{M}}{2}\right)},
$$

then $\lim _{t \rightarrow \infty} \theta_{i j}=0, \forall i, j \in \mathcal{V}$, i.e., the system will reach consensus.

Proof: Let $\boldsymbol{\theta}(t)=\left(\theta_{1}(t), \ldots, \theta_{N}(t)\right)$. The proof is done using the following Lyapunov function

$$
V(\boldsymbol{\theta})=\sum_{i \in \mathcal{V}} \sum_{j \in \mathcal{V}} \frac{1}{2}\left(\theta_{j}-\theta_{i}\right)^{2}=\sum_{i \in \mathcal{V}} \sum_{j \in \mathcal{V}} \frac{1}{2} \theta_{i j}^{2} \geq 0 .
$$

If we compute the derivative of $V$ we obtain

$$
\dot{V}=\sum_{i \in \mathcal{V}} \sum_{j \in \mathcal{V}}\left(\theta_{j}-\theta_{i}\right)\left(w_{j}-w_{i}\right) .
$$

We proceed to show that the derivative is negative if $\theta_{i j} \neq 0$. First, by developing (8) we obtain

$$
\dot{V}=2 N \sum_{i \in \mathcal{V}} \theta_{i} w_{i}-\sum_{i \in \mathcal{V}} \theta_{i} \sum_{j \in \mathcal{V}} w_{j}-\sum_{i \in \mathcal{V}} w_{i} \sum_{j \in \mathcal{V}} \theta_{j},
$$

which by the second point of Lemma 3.1 is simplified to

$$
\dot{V}=2 N \sum_{i \in \mathcal{V}} \theta_{i} w_{i} .
$$

Now, regrouping the terms yields

$$
\sum_{i \in \mathcal{V}} \theta_{i} w_{i}=K \sum_{i \in \mathcal{V}} \theta_{i} \sum_{j \in \mathcal{N}_{i}} w_{i j}=\frac{K}{2} \sum_{(i, j) \in \mathcal{E}}\left(\theta_{i}-\theta_{j}\right) w_{i j} .
$$


Therefore, the derivative of $V$ can be expressed as

$$
\dot{V}=-K N \sum_{(i, j) \in \mathcal{E}} \theta_{i j} w_{i j}
$$

We show now that, under the conditions stated in the theorem, the product $\theta_{i j} w_{i j}$ is positive for all $(i, j) \in \mathcal{E}$. Let us first suppose that $\theta_{i j}>0$. We divide the analysis in four cases. The first two cases consider positive bearing angles:

- Let $\psi_{i j}$ be positive and satisfying $\psi_{i j} \geq \theta_{i j}$. In this case $e_{i j}>e_{j i} \geq 0$. Since both epipoles have the same sign, using the third point of Lemma 3.1 and eq. (3), $0<$ $d_{i j}<\pi / 2$ and then $w_{i j}>0$. Note that this case does not depend on the selection of $\beta$, provided that it has the same sign as $\alpha$.

- If $\theta_{i j}>\psi_{i j} \geq 0$ then $e_{j i}<0 \leq e_{i j}$, which implies that $d_{i j} \geq 0$. However, if $d_{i j}>\pi / 2$, then $w_{i j}<0$ and the control may not be stable. In order to have $w_{i j}>0$ it must hold that $d_{i j} \leq \pi / 2$, which is equivalent to say that $\tan \left(d_{i j}\right)>0$, therefore, using (2) and (3),

$$
\tan \left(d_{i j}\right)=\frac{\frac{\alpha}{\beta}\left(\tan \left(\psi_{i j}\right)-\tan \left(\psi_{i j}-\theta_{i j}\right)\right)}{1+\frac{\alpha^{2}}{\beta^{2}} \tan \left(\psi_{i j}\right) \tan \left(\psi_{i j}-\theta_{i j}\right)}>0 .
$$

The numerator in (10) is always positive due to the conditions on $\theta_{i j}$ and $\psi_{i j}$. Then, to satisfy (10) it is required that

$$
1+\frac{\alpha^{2}}{\beta^{2}} \tan \left(\psi_{i j}\right) \tan \left(\psi_{i j}-\theta_{i j}\right)>0
$$

thus

$$
\frac{\alpha}{\beta}<\sqrt{\frac{1}{\tan \left(\psi_{i j}\right) \tan \left(\theta_{i j}-\psi_{i j}\right)}},
$$

which depends on the ratio $\alpha / \beta$. A lower bound of the right side of eq. (11) is provided later in the proof.

Let us now analyze the cases of negative bearing angles:

- Let us consider first $\psi_{i j}<0$ and $\theta_{i j}-\psi_{i j}<\pi / 2$. When this situation happens $e_{j i}<e_{i j}<0$ and $w_{i j}>0$ because of the third point of Lemma 3.1. Again, when the robots are in this configuration, the control does not depend on $\beta$.

- The last case to analyze appears when $\psi_{i j}<0$ and $\theta_{i j}-$ $\psi_{i j}>\pi / 2$. In this situation the epipoles have different sign, with $e_{i j}<0<e_{j i}$, which implies that, in order to have $w_{i j}>0$, it must happen that $d_{i j}<-\pi / 2$. In other words, $\tan \left(d_{i j}\right)>0$. Now, the numerator in (10) is always negative, which requires

$$
1+\frac{\alpha^{2}}{\beta^{2}} \tan \left(\psi_{i j}\right) \tan \left(\psi_{i j}-\theta_{i j}\right)<0
$$

in order to fulfill (10), and then

$$
\frac{\alpha}{\beta}>\sqrt{\frac{1}{\tan \left(\psi_{i j}\right) \tan \left(\theta_{i j}-\psi_{i j}\right)}} .
$$

Note that (11) and (12) are not in conflict because they are evaluated in different ranges of $\psi_{i j}$. An upper bound of the right side of eq. (12) is provided later in the proof.
The analysis when $\theta_{i j}<0$ can be done taking into account the first point of Lemma 3.1. Noting that $-\theta_{i j}=\theta_{j i}>0$, then $w_{j i}>0$ and $w_{i j}<0$. The system is in equilibrium when $\theta_{i j}=0, \forall(i, j) \in \mathcal{E}$, but due to the fact that the communication graph is connected, then the set of equilibrium points is $\theta_{i j}=$ $0, \forall i, j \in \mathcal{V}$.

We compute now the bounds that satisfy (11) and (12). Let

$$
\gamma\left(\theta_{i j}, \psi_{i j}\right)=\sqrt{\frac{1}{\tan \left(\psi_{i j}\right) \tan \left(\theta_{i j}-\psi_{i j}\right)}} .
$$

We analyze (13) in the intervals $\mathcal{I}_{1}$ and $\mathcal{I}_{2}$

$$
\begin{aligned}
& \mathcal{I}_{1}=\left\{\left(\theta_{i j}, \psi_{i j}\right) \mid 0<\psi_{i j}<\theta_{i j}<\theta_{M}\right\}, \\
& \mathcal{I}_{2}=\left\{\left(\theta_{i j}, \psi_{i j}\right) \mid \psi_{i j}<0<\theta_{i j}<\theta_{M}, \psi_{i j}-\theta_{i j}<-\frac{\pi}{2}\right\},
\end{aligned}
$$

The partial derivative of (13) with respect to $\theta_{i j}$ is equal to

$$
\frac{\partial \gamma}{\partial \theta_{i j}}=\frac{-1}{2 \gamma \tan \left(\psi_{i j}\right) \tan ^{2}\left(\theta_{i j}-\psi_{i j}\right) \cos ^{2}\left(\psi_{i j}-\theta_{i j}\right)} .
$$

We can see that the sign of (14) depends only on the sign of $\tan \left(\psi_{i j}\right)$, which is positive on $\mathcal{I}_{1}$ and negative on $\mathcal{I}_{2}$. Therefore, the function is decreasing with $\theta_{i j}$ on $\mathcal{I}_{1}$ and increasing on $\mathcal{I}_{2}$ and in both cases the bound we are looking for will be achieved in $\theta_{i j}=\theta_{M}$.

If we compute the derivative of (13) with respect to $\psi_{i j}$, already considering $\theta_{i j}=\theta_{M}$ we obtain

$$
\frac{\partial \gamma}{\partial \psi_{i j}}=\frac{\sin \left(\theta_{M}-\psi_{i j}\right) \cos \left(\theta_{M}-\psi_{i j}\right)-\sin \left(\psi_{i j}\right) \cos \left(\psi_{i j}\right)}{2 \gamma \sin ^{2}\left(\theta_{M}-\psi_{i j}\right) \sin ^{2}\left(\psi_{i j}\right)} \text {. }
$$

The only minimum of (15) on the interval $\mathcal{I}_{1}$ is in $\psi_{i j}=$ $\theta_{M} / 2$. The maximum on $\mathcal{I}_{2}$ is found on the value $\psi_{i j}=$ $-\pi / 2+\theta_{M} / 2$. Using trigonometry equivalences we obtain that

$$
\gamma\left(\theta_{M},-\pi / 2+\theta_{M} / 2\right)=\frac{1}{\gamma\left(\theta_{M}, \theta_{M} / 2\right)} .
$$

Finally, by noting that $\gamma\left(\theta_{M}, \theta_{M} / 2\right)=1 / \tan \left(\theta_{M} / 2\right)$, the condition in (6) is obtained.

The last point to check is the invariance of the set $\left|\theta_{i j}\right| \leq$ $\theta_{M}, \forall i, j$. To show this, let us consider a fixed reference frame $\mathcal{F}$, and let $\theta_{\max }$ and $\theta_{\min }$ be the maximum and minimum orientation values in such frame, both of them positive. We show that the value $\left(\theta_{\max }-\theta_{\min }\right)^{2}=\theta_{\min \max }^{2}$ is decreasing over time. The derivative of this quantity is equal to $2 \theta_{\min \max }\left(w_{\max }-w_{\min }\right)$, where $\theta_{\min \max }>0$. Now, let us note that $\theta_{\max i}=\theta_{i}-\theta_{\max } \leq 0$, and $\theta_{\min i}=\theta_{i}-\theta_{\min } \geq 0$ for all $i$ and all $t$. Therefore, taking into account that we have shown by eq. (9) that the product $\theta_{i j} w_{i j}$ is positive for all $i$ and $j$, we deduce that $w_{\max } \leq 0$ and $w_{\min } \geq 0$, and the invariance is shown.

Remark 3.1: We did not consider in the proof the special cases $\psi_{i j}=0, \psi_{i j}=\pi / 2, \psi_{i j}=\theta_{i j}$ and $\psi_{i j}-\theta_{i j}= \pm \pi / 2$ to compute the bounds (11) and (12). For these values the derivatives (14) and (15) are not defined and by multivariate analysis [31], there might be an extreme in these points. However, it can be shown by straight calculations that the right hand side of (9) is negative when $\theta_{i j} \neq 0$ for each of the special cases independently of $\beta$. 
Remark 3.2: The controller can also be used to make the robots reach consensus with respect to a "leader" robot. If the leader is headed to the desired fixed orientation $\theta^{d}$, the followers apply the proposed controller and the conditions of Theorem 3.2 are satisfied, then eventually $\theta_{i j}=0$ for all pair of robots. Since the leader has not changed its orientation, we conclude that the orientations of all the robots must be equal to $\theta^{d}$.

An additional advantage of our controller, based on nearest neighbor rules, is the natural robustness against the initial lack of shared information between some pairs of robots. If two neighbors initially cannot compute their epipoles due to the lack of enough matches, as long as there is an alternative path in the graph (of arbitrarily length) that communicates these two robots, then the whole team will still reach the consensus.

\section{A. Influence of $\beta$}

Let us note that Theorem 3.2 provides more information than just a condition to reach the consensus. It also provides a relation between the calibration knowledge and the relative orientation between the agents. The relationship between $\beta$ and $\alpha$ given in eq. (6) suggests that the calibration needs to be known to guarantee convergence to the consensus. However, let us note that one could assign a value to $\beta$ without any knowledge of $\alpha$ (or a corrupted value of $\alpha$ ) and still reach the consensus. The closer it is $\beta$ to the real focal length, $\alpha$, the larger the value of $\theta_{M}$ is allowed to be, but always upper bounded by $\pi / 2$. Regarding this bound it is interesting to point out that, since monocular cameras usually have a limited field of view of about 90 degrees, even if our analysis did not require it, the need of sharing common observations of the environment to reach the consensus would in practice impose the same limitation.

On the other hand is the issue of how the time to reach consensus is affected by a particular choice of $\beta$. The following proposition shows how $w_{i j}$ is affected by variations of $\beta$.

Proposition 3.3: If the conditions of Theorem 3.2 hold, then the relationship between $w_{i j}$ and $\beta$ is not monotonic.

Proof: The derivative of $d_{i j}$ in eq. (3) with respect to $\beta$ is equal to

$$
\frac{\partial d_{i j}}{\partial \beta}=\frac{\left(e_{i j} e_{j i}-\beta^{2}\right)\left(e_{i j}-e_{j i}\right)}{\left(e_{i j}^{2}+\beta^{2}\right)\left(e_{j i}^{2}+\beta^{2}\right)} .
$$

The sign of the derivative does not depend on the denominator, therefore, we only analyze the sign of the numerator. Similarly to what we did in the proof of Theorem 3.2 we divide the analysis in different cases. Specifically we choose the cases when $\theta_{i j}>\psi_{i j} \geq 0$ and $\psi_{i j}<0$ and $\theta_{i j}-\psi_{i j}>\pi / 2$.

In the first case $e_{j i}<0 \leq e_{i j}$ which implies that the sign of (17) is negative. Considering that in this case $w_{i j}=d_{i j}$, then $w_{i j}$ is a decreasing function of $\beta$.

On the other hand, in the second case $e_{i j}<0<e_{j i}$ and the sign of (17) is positive. In this case $d_{i j}<-\pi / 2, w_{i j}=\pi+d_{i j}$ and therefore $w_{i j}$ is increased when we increase $\beta$.

We can use the previous proposition to analyze the convergence time to consensus. Let us first consider the case of two robots, $i$ and $j$. Recall that when $\beta=\alpha$ then $w_{i j}=\theta_{i j}$, and our controller is linear. Now suppose the case where $w_{i j}$ is decreased with $\beta$, therefore by increasing $\beta$ we reach that $w_{i j}<\theta_{i j}$. This could be seen as a modification of the control law (5) considering a smaller gain. By having a smaller gain, the robots will require more time to reach consensus.

If we consider the case where $w_{i j}$ is increased with $\beta$, we can do the same analysis to realize that in this situation the time required to reach the consensus is going to be smaller. The case of $N$ robots can be analyzed by a straightforward extension of the case of two robots. Letting each pair of robots to be initially aligned in such a way that $w_{i j}$ is decreased (or increased) the same analysis holds. Therefore, there are some situations in which it is desirable to choose $\beta>\alpha$ to ensure that the robots will reach the consensus in the least time whereas in other cases it is better to choose $\beta<\alpha$. Since this relationship can be tight to the epipoles, the robots could use them to try to speed up the convergence to the consensus by a proper selection of the parameter $\beta$.

Regarding the final orientation of the robots, the only scenario where we can say with precision the consensus value is when $\beta=\alpha$, i.e., a perfectly calibrated scenario. In such case our controller becomes linear and follows the guidelines of [1], converging to the average of the initial orientations.

\section{ROBUSTNESS OF THE CONTROLLER TO MORE REALISTIC CONDITIONS}

The controller presented in the previous section is only valid for a fixed communication topology. However, under more realistic conditions it is most likely that the communication topology will change as the robots move, or that the calibration of the cameras is not entirely equal for all the robots. In this section we analyze some of the issues that might appear in more realistic conditions, and study the robustness of the proposed controller.

\section{A. Analysis Under Switching Topologies}

There are multiple reasons to study the robustness of the controller against changes in the communication topology. The most usual reason comes from the motion of the robots, but the use of visual sensors introduce other issues that can also be modeled as changes in the topology like perception issues and computational demands:

- Changes in the topology due to perception issues: It is possible that two neighboring robots cannot compute their epipoles due to blurry images or temporal occlusions, which would be the same as assuming that they are not neighbors in the communication graph.

- Changes in the topology for computational demands: The number of neighbors determines the amount of time each robot will require to compute its control input, because for each neighbor a computation of the epipoles using a robust algorithm, e.g., DLT+RANSAC [30], is required. A subset of the robots can be selected at each time to reduce the computational time of each robot, which is equivalent to consider a time-varying topology.

For these reasons it is interesting to analyze the controller in the presence of changes in the communication topology. For 
simplicity, we will leave out of the analysis the dependency of state in the changes in the topology, considering instead that these changes occur at arbitrary times, satisfying the following standard assumptions for this case [1], [9]:

Assumption 4.1: There exists a lower bound, $\delta>0$, on the time between two consecutive changes in the topology. Denoting $t_{k}, k \in \mathbb{N}$, the discrete time instants when the topology changes, then $t_{k+1}-t_{k} \geq \delta, \forall k$.

Assumption 4.2: There exists a positive time period $T$ such that, for any instant of time, $t$, the collection of communication topologies in the time interval $(t, t+T)$ is jointly connected.

The following result states that the proposed controller can handle changes in the communication topology, reaching the desired agreement in the headings of the robots.

Proposition 4.1: Consider a robotic network which satisfies the conditions stated in Theorem 3.2 and Assumptions 4.1 and 4.2. If the robots use the control law

$$
w_{i}=K \sum_{j \in \mathcal{N}_{i}(t)} w_{i j}
$$

then $\lim _{t \rightarrow \infty} \theta_{i j}=0, \forall i, j \in \mathcal{V}$.

Proof: First of all, using the same arguments as in the proof of Theorem 3.2, it is easily verifiable that the set of relative orientations $\theta_{i j}$ is invariant.

Now we use again the Lyapunov function defined in (7). The new derivative of $V$ is

$$
\dot{V}=-K N \sum_{(i, j) \in \mathcal{E}(t)} \theta_{i j} w_{i j} \leq 0
$$

and the function is a weak common Lyapunov function for all $\mathcal{E}(t)$, and therefore, for any network topology. Combining this with Assumption 4.1, we can assert that the system is stable [32].

Denote $\boldsymbol{\theta}^{*}$ as the set of points with all the orientations equal, i.e., $\boldsymbol{\theta}^{*}=\left\{\boldsymbol{\theta} \mid \theta_{i j}=0, \forall i, j \in \mathcal{V}\right\}$. Let $\mathcal{G}_{N}$ be the set of graphs composed by $N$ nodes

$$
\sum_{\mathcal{G} \in \mathcal{G}_{N}} \dot{V}_{\mathcal{G}}(\boldsymbol{\theta})<0
$$

for all $\boldsymbol{\theta} \notin \boldsymbol{\theta}^{*}$. Therefore $V$ is a common joint Lyapunov function of the system [33], [34], and $\boldsymbol{\theta}^{*}$ are the only equilibrium points that all the graphs have in common. The ergodicity requirement on the switching signal is found in Assumption 4.2. Therefore, we conclude that the robots will converge to some $\boldsymbol{\theta} \in \boldsymbol{\theta}^{*}$ and the consensus will be reached.

This robustness against changes in the communication topology can be used by the robots to reduce the computational demands required to obtain all the pairs of epipoles corresponding to all the neighbors. We can let the robots select a subset of their neighbors at each time step in such a way that Assumption 4.2 is satisfied, ensuring convergence to the consensus with a smaller computational cost. In the following we present the two distributed strategies considering the subsets to be composed by a single neighbor, though its extension to several neighbors is straightforward.
1) Strategy 1: Choose the neighbor that was not selected for the longest time: The first strategy we propose consists of selecting at each iteration the neighbor that was not selected for the longest time. Let each robot handle a vector $\mathbf{N}_{i}(t)=$ $\left[N_{i 1}(t), \ldots, N_{i N}(t)\right]$, with $N_{i j}(t)$ representing the number of communication rounds that has passed since the last time that robot $i$ chose robot $j$ as the selected neighbor to compute the epipoles.

Initially, $N_{i j}(0)=0$ for all $j$. Then, at time $t$, the neighbor selected by robot $i$, denoted by $j(t)$, will be

$$
j(t)=\arg _{j \in \mathcal{N}_{i}} \max N_{i j}(t),
$$

and the control input of the robot

$$
w_{i}(t)=K w_{i j(t)} .
$$

Once the robot has computed the epipoles and the control input, it updates the vector $\mathbf{N}_{i}(t)$ with the following rule

$$
N_{i j}(t+1)=\left\{\begin{array}{ll}
0 & \text { if } j=\{j(t), i\} \\
N_{i j}(t)+1 & \text { otherwise }
\end{array},\right.
$$

so that it ensures that $j(t)$ will not be chosen again until all the other possible neighbors have been chosen once.

Proposition 4.2: Let all the robots select, at each iteration, one neighbor to compute the epipoles using equations (20), (22) and move using the controller in eq. (21) considering only this neighbor. Then, the system will reach the consensus.

Proof: At any iteration, $t$, each robot selects only one of its neighbors, which may lead to a disconnected digraph, $\mathcal{G}(t) \subseteq \mathcal{G}$. However, at different iterations the same robot will select different neighbors in the communication graph Therefore, considering Assumption 4.2, in a worst case scenario the graph

$$
\mathcal{G}(t) \cup \mathcal{G}(t+1) \cup \ldots \cup \mathcal{G}(t+N T)
$$

is again jointly connected, which means that Assumption 4.2 is still satisfied when using this strategy. Considering that the time required to compute the epipoles is not zero, Assumption 4.1 is also satisfied and then we can conclude that the time varying evolution of the graph satisfies the conditions to reach the consensus.

2) Strategy 2: Choose the neighbor with more misalignment: The second strategy we propose is designed to reduce the orientation error with the neighbor that supposedly is farthest away at each iteration.

Let each robot have a vector $\hat{\mathbf{d}}_{i}(t)=\left[\hat{d}_{i 1}(t), \ldots, \hat{d}_{i N}(t)\right]$, with $\hat{d}_{i j}(t)$ being the last value of $d_{i j}$ computed by robot $i$ using the information provided by robot $j$, equation (3). Initially, $\hat{d}_{i j}(0)=\infty$ for all $j$. The neighbor selected at each iteration is chosen by

$$
j(t)=\arg _{j \in \mathcal{N}_{i}(t)} \max \hat{d}_{i j}(t),
$$

that is, the one with the highest forecasted misalignment at current time. The control input of each robot is then assigned as in eq. (21).

With the epipoles computed, the update of $\hat{\mathbf{d}}_{i}(t)$ executed in this case is

$$
\hat{d}_{i j}(t+1)=\left\{\begin{array}{ll}
d_{i j} & \text { if } j=j(t) \\
\hat{d}_{i j}(t) & \text { otherwise }
\end{array},\right.
$$


with $d_{i j}$ the value computed in equation (3).

Proposition 4.3: Let all the robots select, at each iteration, one neighbor to compute the epipoles using equations (23), (24) and move using the controller in eq. (21) considering only this neighbor. Then, the system will reach the consensus.

Proof: With this strategy we cannot ensure that the robots are changing the selected neighbors at each iteration. However, we know that if a robot $i$ does not change the neighbor, $j$, eventually, it tends to align with it, meaning that $d_{i j} \rightarrow 0$ and so does $\hat{d}_{i j}$. Therefore, at some point there will be some $k \in \mathcal{N}_{i}$ such that $\hat{d}_{i j}>\hat{d}_{i k}$ and the robot will change the selected neighbor. Using again the same argument we know that eventually all the neighbors will be selected and using the same arguments as in Proposition 4.2 we conclude that the system will reach the consensus.

There are several advantages of using any of the two proposed strategies instead of computing the epipoles with the images sent by all the neighbors. First of all, the consensus is achieved requiring less computations at each communication round. Each pair of computed epipoles requires an initial step to match the features of the two images plus a robust method to estimate the epipolar constraint. By selecting only one neighbor, we are executing this step only once at each iteration instead of $\left|\mathcal{N}_{i}\right|$ times. This computational reduction can be of high interest in situations where the energy of the robots is limited.

Synchronization issues are also solved. Note that the controller requires the images of all the robots to be acquired (approximately) at the same instant. If each robot does not acquire a new image until it has processed all the received information, and assuming that the number of neighbors is not going to be the same for all the robots, then without additional mechanisms to synchronize the network there will appear time discrepancies among the matched images. On the other hand, making all the robots to select only one neighbor to compute the epipoles will imply similar computation times for all of them, leading to a natural synchronization.

\section{B. Cameras with different calibrations}

When modeling the team of robots in section II we assumed that all the robots wore the same camera with the same intrinsic parameters, i.e., the parameter $\alpha$ was the same for all the robots.

In a real scenario, even if all the robots are equipped with the same camera (same model, same characteristics, etc.), it is very unlikely that all the calibrations of the $N$ cameras are exactly the same. Let us consider that each robot is wearing a different camera with a different calibration. We denote by $\alpha_{i}$ the calibration parameter of robot $i$. If the parameter $\beta$ in (3) is kept at the same value for all the robots, then the final configuration of the network will not be the desired consensus because equal epipoles do not imply equal orientations anymore. The following proposition gives the direct expression of the final error between the orientation of pairs of robots:
Proposition 4.4: The error in the orientation is equal to

$$
\tilde{\theta}_{i j}=\arctan \left(\frac{\left(\alpha_{j}-\alpha_{i}\right) \sin \psi_{i j} \cos \psi_{i j}}{\alpha_{i} \sin ^{2} \psi_{i j}+\alpha_{j} \cos ^{2} \psi_{i j}}\right)
$$

Proof: The consensus is achieved when $e_{i j}=e_{j i}$. This implies

$$
\arctan \left(\frac{\alpha_{i}}{\beta} \tan \psi_{i j}\right)=\arctan \left(\frac{\alpha_{j}}{\beta} \tan \left(\psi_{i j}-\theta_{i j}\right)\right) .
$$

Developing the equality yields

$$
\alpha_{i} \frac{\sin \psi_{i j}}{\cos \psi_{i j}}=\alpha_{j} \frac{\sin \psi_{i j} \cos \theta_{i j}-\cos \psi_{i j} \sin \theta_{i j}}{\cos \psi_{i j} \cos \theta_{i j}+\sin \psi_{i j} \sin \theta_{i j}} .
$$

Rearranging the terms we obtain

$$
\begin{aligned}
& \left(\alpha_{j}-\alpha_{i}\right)\left(\sin \psi_{i j} \cos \psi_{i j}\right) \cos \theta_{i j}= \\
& \left(\alpha_{i} \sin ^{2} \psi_{i j}+\alpha_{j} \cos ^{2} \psi_{i j}\right) \sin \theta_{i j} .
\end{aligned}
$$

Thus

$$
\frac{\sin \theta_{i j}}{\cos \theta_{i j}}=\frac{\left(\alpha_{j}-\alpha_{i}\right) \sin \psi_{i j} \cos \psi_{i j}}{\left(\alpha_{i} \sin ^{2} \psi_{i j}+\alpha_{j} \cos ^{2} \psi_{i j}\right)} .
$$

and taking the arc tangent (25) is obtained.

Equation (25) shows that the final error in the orientation of the robots depends not only on the difference between their calibrations but also on their relative bearing angle. This is good news because we can make the robots achieve the desired configuration, even if they do not have the same cameras.

Corollary 4.5: If the robots are in parallel or leader follower formations, i.e., $\psi_{i j}=\pi / 2$ or $\psi_{i j}=0$, then the consensus is achieved.

The corollary is proved just by replacing these two values of $\psi_{i j}$ in eq. (25) and seeing that the error value is always zero.

Nevertheless, in the experiments section we show the robustness of the controller to different cameras, even if the bearing angle between pairs of robots is different to $\pi / 2$ or zero.

\section{Simulations}

In this section, the properties of the proposed controller are shown in simulations. The experiments have been carried out using Matlab. As an illustrative example, let us consider a robotic network composed by ten robots with initial positions and orientations depicted in Fig. 2 and communications defined by the dashed lines. To simulate the vision system we have randomly generated a set of $3 \mathrm{D}$ features in the environment. The cameras have calibration matrix $\mathbf{K}=$ $\operatorname{diag}(300,300,1)$, and a resolution of $640 \times 480$ pixels. This implies that the robots have a limited field of view of 94 degrees. Under these conditions not all the robots can observe each other in their images. For example, robot two can only communicate with robot four and there are no other robots visible in its field of view. However, using the epipoles it can compute a control input to align its heading with the one of robot four.

The results of using the proposed controller with $\beta=250$ are shown in Figs. 2 and 3. Since the maximum relative orientation between a pair of robots is 1.23 the bounds on $\beta$ required to converge are $212<\beta<423$ (eq. (6)) and in this case the controller reaches the consensus. The right plot 

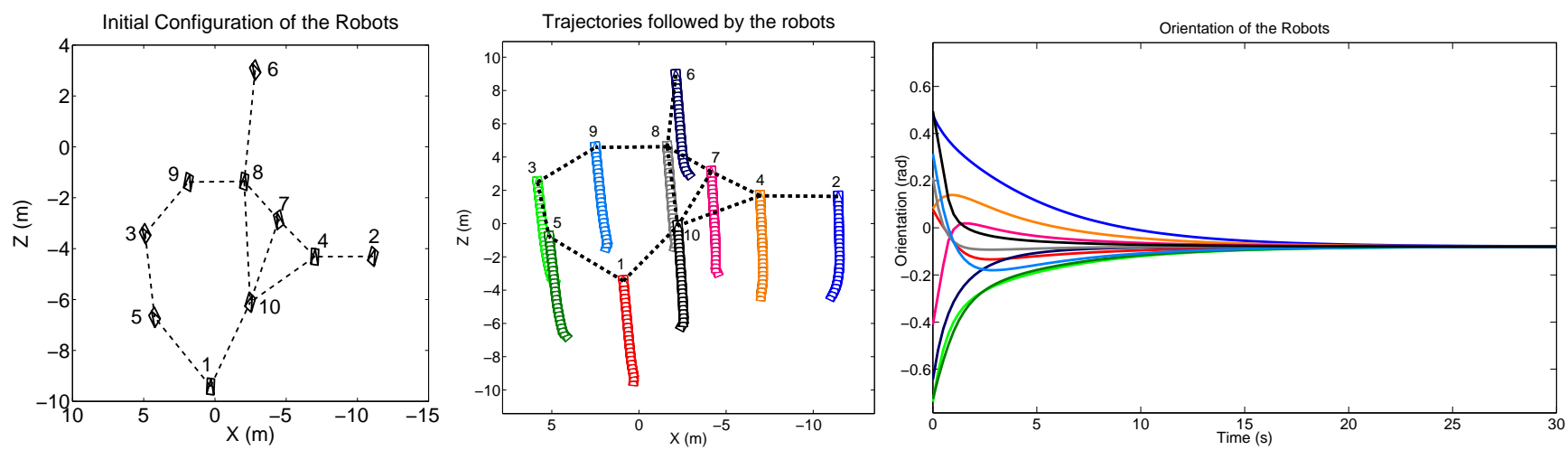

Fig. 2: Initial positions (left) and trajectories followed by the robots (middle). Dashed lines represent direct communications between robots. In the right figure we can observe the values of the orientation of the robots, which eventually reach the consensus.
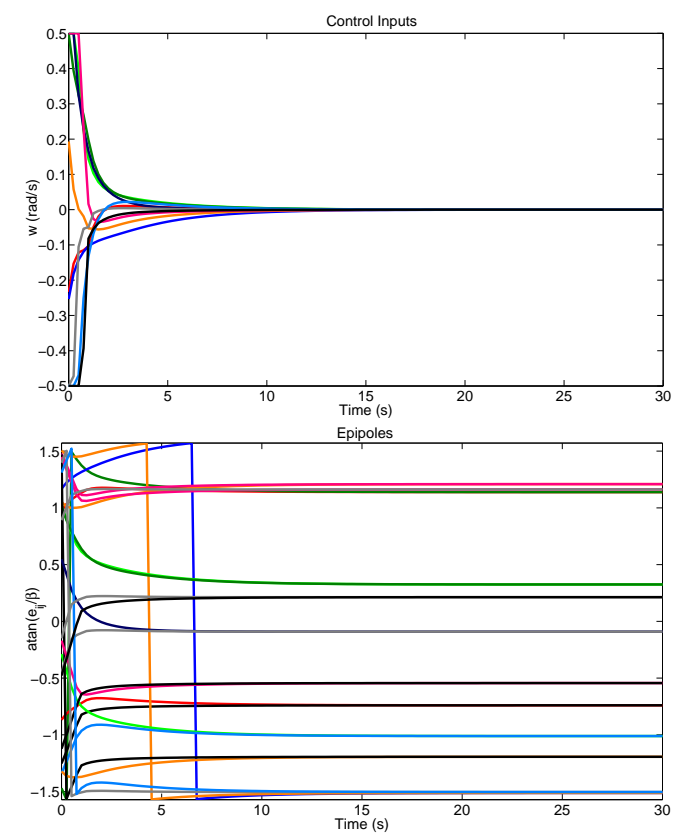

Fig. 3: Control inputs and evolution of the pairs of computed epipoles for the robotic network in Fig. 2.

in Fig. 2 shows the evolution of the orientation of the robots, which converge to the same value for all of them. The top graphic in Fig. 3 depicts the control inputs and the bottom graphic the evolution of all the pairs of computed epipoles.

\section{A. Influence of noise and calibration}

It is interesting to analyze the robustness of the controller to the different parameters that take part in the process such as the calibration of the cameras or the noise of the features used to compute the epipoles. To do so, we have run Monte Carlo simulations to extract statistic measurements about the robustness of our approach. For a better understanding, in these experiments we measure the results using degrees instead of radians. We have considered 1000 configurations of ten robots with random initial positions and random orientations within the interval $\left(-45^{\circ}, 45^{\circ}\right)$. As in the previous simulation $\alpha$ has been set equal to 300 and $\beta$ equal to 250 . The initial mean error of the 1000 trials is equal to $73.99^{\circ}$ with a standard deviation of $9.64^{\circ}$. Then we have run our controller considering different scenarios. In the first one we have added gaussian noise to the features viewed by the robots. The second and the third scenario have considered different calibration of the cameras, varying the focal length and the principal point respectively.

The aggregate results of all the experiments are shown in Table I. For each trial we have measured the mean final misalignment $\left(\theta_{\max }-\theta_{\min }\right)$ with the standard deviation after running the controller for 30 seconds. Using the initial mean error in the orientation we have included an additional row with the relative final error (in percentage). The first column is the same in all the cases and represents the ideal scenario with no noise or different calibrations. In the perfect scenario the robots always achieve consensus with a negligible error $\left(0.77^{\circ}\right)$. As the noise in the images is increased, the final error in the orientations of the robots reaching a final mean error of $6.7666^{\circ}$ for standard deviation of 2 pixels in the noise of the features. Regarding different calibrations, the controller seems to be more robust to different focal length than to different principal point of the cameras. Nevertheless, for vastly large levels of noise (50 pixels of standard deviation) the relative error with respect to the initial configuration remains bounded ( $11.15 \%$ and $14.85 \%$ respectively).

\begin{tabular}{|l|c|c|c|c|}
\hline \multicolumn{5}{|c|}{ Image noise } \\
\hline Features Std. dev (pixels) & 0.0 & 0.5 & 1.0 & 2.0 \\
Consensus Mean error (deg) & 0.7786 & 3.8847 & 5.0134 & 6.7666 \\
Consensus Std. dev (deg) & 2.1301 & 5.1795 & 5.5119 & 6.2968 \\
Relative error (\%) & 1.05 & 5.25 & 6.77 & 9.14 \\
\hline \multicolumn{5}{|c|}{ Different focal length $(\alpha)$} \\
\hline$\alpha$ Std. dev (pixels) & 0 & 10 & 25 & 50 \\
Consensus Mean error (deg) & 0.7786 & 3.1283 & 4.9618 & 8.2506 \\
Consensus Std. dev (deg) & 2.1301 & 4.8243 & 4.8128 & 5.2712 \\
Relative error (\%) & 1.05 & 4.22 & 6.70 & 11.15 \\
\hline \multicolumn{5}{|c|}{ Different principal point } \\
\hline Ppal. Point Std. dev (pixels) & 0 & 10 & 25 & 50 \\
Consensus Mean error (deg) & 0.7786 & 2.5826 & 5.7934 & 10.9941 \\
Consensus Std. dev (deg) & 2.1301 & 4.6947 & 5.2249 & 6.8346 \\
Relative error (\%) & 1.05 & 3.49 & 7.83 & 14.85 \\
\hline
\end{tabular}

TABLE I: Robustness of the controller to noise and different calibrations

In order to corroborate the result in Proposition 3.3 we have analyzed the time required to reach consensus depending on $\beta$. We have run a simulation considering again the 1000 initial random scenarios and we have computed the time required to reach the consensus (with a tolerance of $10^{-2} \mathrm{rad}$ ) for different values of $\beta$. The results have been included in Table II. The most significative information of the table is the large standard deviation which corroborates that, for the same value of $\beta$ 
depending on the configuration of the robots sometimes we obtain large convergence time values whereas other we obtain small ones.

\begin{tabular}{|l|c|c|c|c|c|}
\hline \multicolumn{5}{|c|}{ Convergence times $(\alpha=300)$} \\
\hline$\beta$ & 200 & 250 & 300 & 350 & 400 \\
Mean time (s) & 36.10 & 32.57 & 32.19 & 33.27 & 34.93 \\
Std. dev (s) & 30.60 & 28.94 & 28.92 & 29.95 & 31.01 \\
\hline
\end{tabular}

TABLE II: Time required to reach the consensus in function of $\beta$

\section{B. Simulations in a virtual scenario}

In the last simulation we have considered a virtual scenario. Using the virtual reality toolbox of MatLab we have created a virtual world. In this way, the robots acquire virtual images of resolution $640 \times 480$ pixels depending on their position and orientation. In this case the robots need to use real computer vision algorithms to estimate the epipoles. The aim of using virtual images is that we can check the performance of all the aspects of our proposal against the ground truth. We have extracted SIFT [35] features from the virtual images and the 8 point algorithm with RANSAC [36] to match them in a robust way and to compute the epipoles between pairs of robots. An example of the images acquired by the robots and the features extracted and matched can be found in Fig. 4. Although most of the features are detected in the clouds or other dynamic elements of real scenes, the computation of the epipoles is not affected because each set of images is acquired at the same time, which means that for the robots they represent features of a static scene.

We have considered a fixed robotic network composed by ten robots with initial positions and orientations depicted in Fig. 5 and communications defined by the black lines. As we can see, there are some robots that have up to five neighbors in the communication graph whereas others only have one or two neighbors.

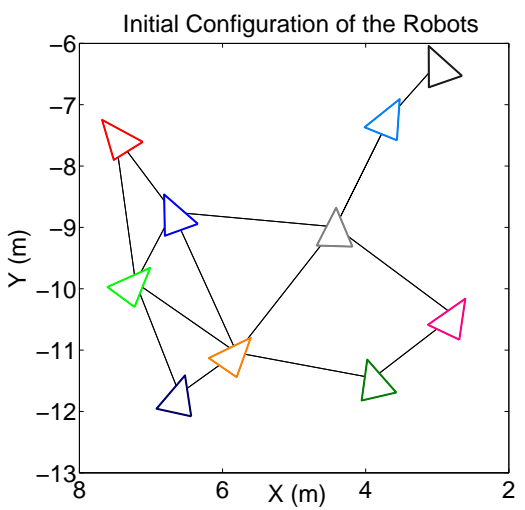

Fig. 5: Initial configuration of the team of robots in the experiments. Black lines represent direct communication between robots.

We have executed the controller in eq. (5) without using a neighbor selection strategy, and therefore with a fixed communication topology, and the controller (21) using the two strategies proposed in section IV, forcing time-varying topologies. The evolution of the orientation of the robots in the three cases can be seen in Fig. 6, in all the cases the robots reach the consensus. The evolution of the orientation when the information of all the neighbors is used is smoother and the consensus is reached in less time. However, the difference with respect to the other two graphics is negligible and in the simulation we are not considering the actual time spent by each robot to compute the epipoles with all the neighbors. The second strategy (neighbor with more misalignment) reaches the consensus relatively faster than the first strategy, which makes sense because it tries to reduce the error with the robot with most misalignment. The control inputs of the robots in each scenario are depicted in Fig. 7. The second strategy also seems to be better than the first one in this aspect.

However, this simulation is not considering the real time spent to compute the inputs. We have measured the time spent to compute the control inputs when the robots do not use the proposed strategies and when they do to point out the real advantages of using a strategy to select a subset of neighbors. The computational time spent by each robot at each iteration is depicted in Fig. 8. We can see that using any of the two strategies the computational time per iteration and robot remains bounded and similar whereas in the standard case there are big variations in the loop time, depending on the number of neighbors of each robot. The statistics of these times are shown in Table III.

\begin{tabular}{|l|c|c|c|}
\hline Quantity & No strategy & Strategy 1 & Strategy 2 \\
\hline Mean time & 12.65 & 3.80 & 3.85 \\
Std. dev & 5.83 & 0.77 & 0.75 \\
Max time & 27.99 & 5.92 & 5.84 \\
Min time & 2.55 & 1.49 & 1.28 \\
\hline
\end{tabular}

TABLE III: Computational time (seconds per robot and iteration)

\section{EXPERIMENTS WITH REAL ROBOTS}

We have also tested our proposal in a real platform. In this section we briefly describe the whole setup, the experiments carried out and we show the results obtained. Nevertheless, for a better visualization of the results we refer the reader to the supplementary videos, where the whole motion of the robots can be seen.

The experiments have been carried out with three robots Pioneer 3Dx inc. with non-holonomic motion constraints like the ones described in eq. (1). Each robot has been equipped with a laptop and a wireless antenna to communicate with the other two robots. Regarding the vision system of the robots, in most of the experiments we have used a Kinect camera onboard of each robot. In order to analyze the robustness of the controller when using different cameras in one of the experiments we have equipped one robot with a Unibrain camera, which in addition to have different intrinsic parameters, it also has the disadvantage of acquiring images with radial distortion. The two cameras can be seen in Fig. 9 and an example of the images acquired by each camera is shown in Fig. 10.
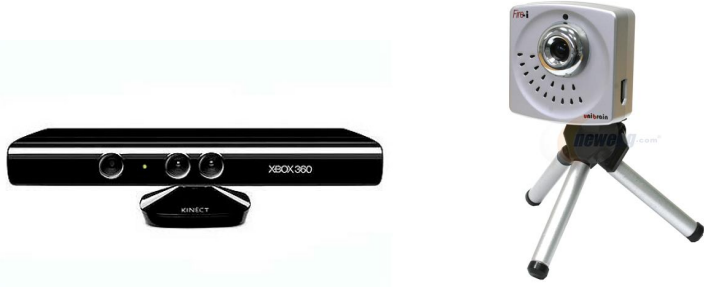

Fig. 9: Cameras equipped by the robots in the experiments. 
Images
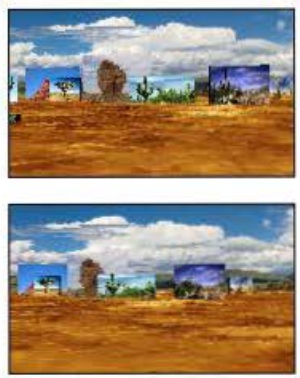
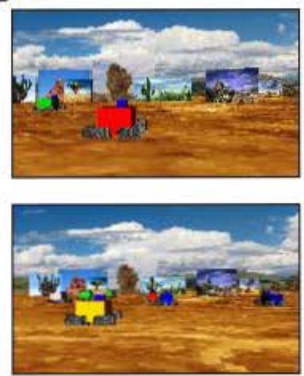

Matches robots 1 and 2

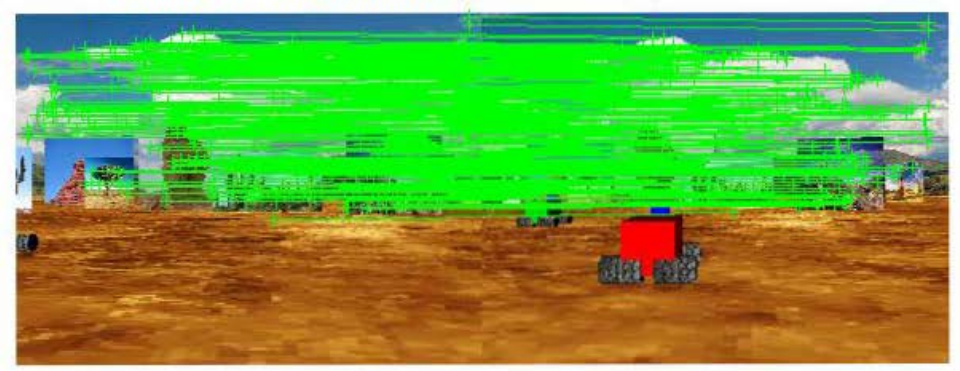

Fig. 4: Images acquired in the virtual environment and matches between the SIFT descriptors. Orientation of the Robots (No strategy)
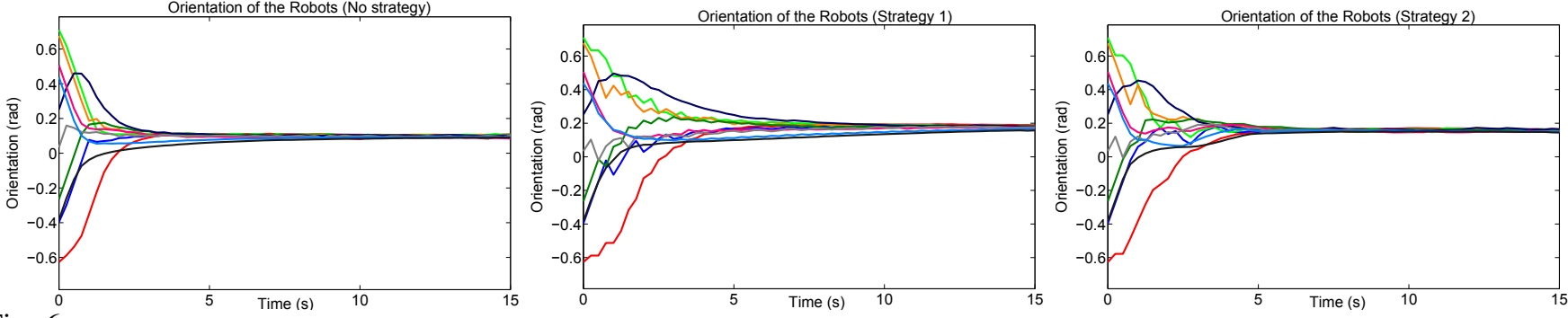

Fig. 6: Orientation of the robots using the distributed controller without any strategy to select the neighbors (left), with the strategy to choose the neighbor that was not selected for the longest time (middle) and with the strategy to choose the neighbor with the most misalignment (right). In the three cases all the robots reach consensus at nearly the same time.
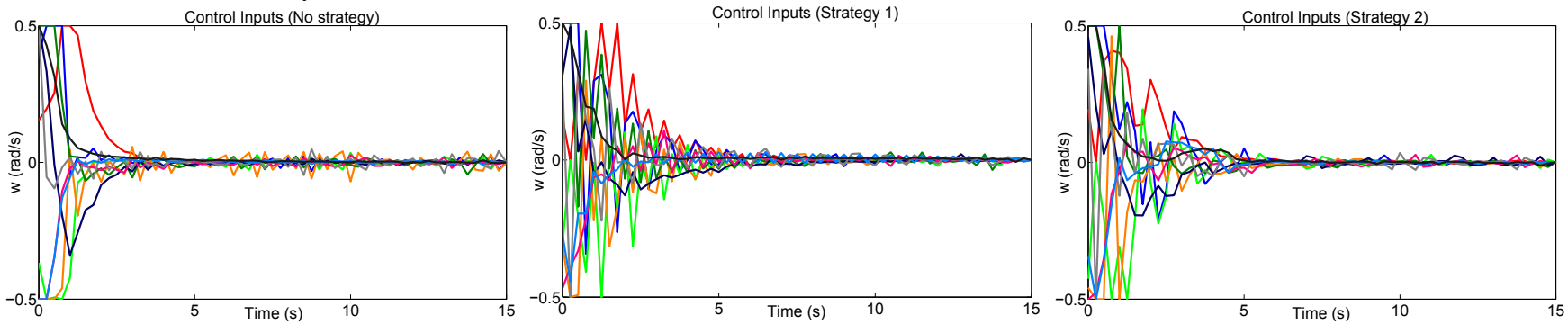

Fig. 7: Control inputs of the ten robots in the three scenarios: without any strategy to select the neighbors (left), with the strategy to choose the neighbor that was not selected for the longest time (middle) and with the strategy to choose the neighbor with the most misalignment (right).
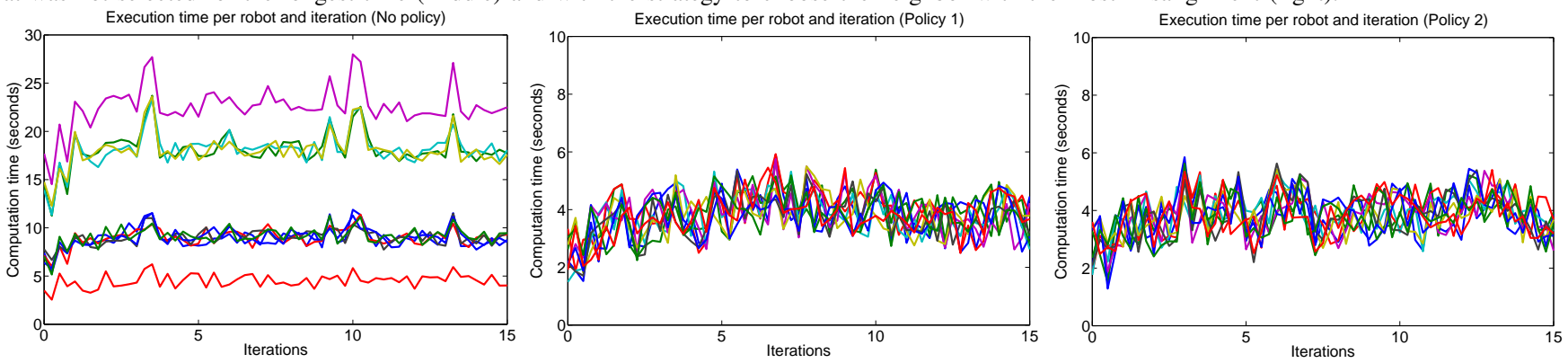

Fig. 8: Computational time spent by each robot at each iteration: without any strategy to select the neighbors (left), with the strategy to choose the neighbor that was not selected for the longest time (middle) and with the strategy to choose the neighbor with the most misalignment (right). As we can see, when the robots use a strategy, all of them spend approximately the same computational time, whereas without a strategy each robot requires a different time depending on its neighbors.
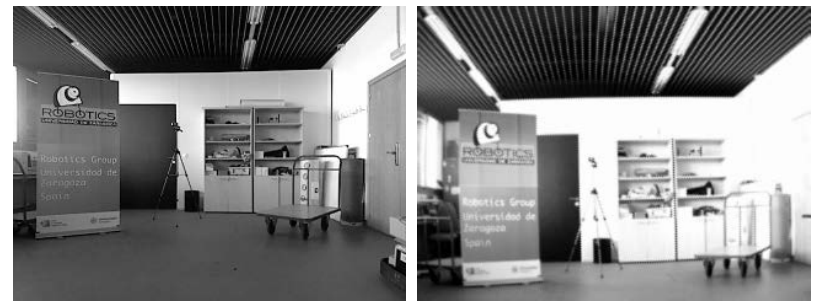

Fig. 10: Example of images captured by the two cameras used in the experiments, Kinect image (left) and Unibrain image (right).

We have used SIFT features and the 8 point algorithm with RANSAC for matching and computing the epipoles. Since in this environment the loop time is important, we have used images of resolution $320 \times 240$ pixels, reducing the time required to extract the SIFT descriptors. In addition, by using smaller images, the number of features was also reduced, which implies less communications between the robots. The drawback of this reduction is that there are some iterations in which the epipoles cannot be computed due to the lack of enough good matches to obtain a robust estimation.

Since there are only three robots, in all the experiments we have considered a fixed communication topology in which every robot can communicate with each other. In order to communicate, the robots have used a real time communication 
protocol [37]. Let us recall that the proposed method assumes some synchronization between the robots, in the sense that the images acquired by all of them should be acquired at the same time. In a real scenario this is difficult to achieve. For that reason we have used a barrier scheme in which the robots do not acquire a new image until the previous one has been processed. The scheme followed by the robots at each iteration is described next:

- Acquire an image and extract the SIFT descriptors.

- Communicate the descriptors to the other robots.

- Receive the SIFT extracted from the other two robots.

- Match the received features with the extracted ones and compute the epipoles.

- Compute the control velocity from the epipoles and send an ACK message to the other robots to acquire a new image.

- Wait until the reception of the ACK of the other robots and begin with the whole process again.

Although with this approach there are still some time gaps between some of the frames of the robots, they are negligible in the final results. The total time for each iteration is around 2 seconds and most of the time is used in communicating the approximately 300 SIFT descriptors extracted per image (around $300 \mathrm{~KB}$ ). We have not dealt with packet drops, as this is handled by the communication protocol. In the following we describe the experiments carried out:

1) Experiment outdoors: In the first experiment we have taken the robots to the parking lot of the Escuela de Ingeniería y Arquitectura of the Universidad de Zaragoza. The robots move with constant linear velocity of $0.1 \mathrm{~m} / \mathrm{s}$. The initial and final configuration of the robots can be seen in Fig. 11. Since there is no common frame to measure the global orientation of the robots, we cannot offer ground truth results about the error in the initial and the final orientation. Nevertheless, in the figure we can see that the robots end up in a configuration with the same orientation (up to an acceptable error). Let us remark that although the robots that are behind can see the third robot in their images, this information is not used by the control law. The robot that does not see other robots in its image is also turning with the epipoles, as can be seen comparing its initial and final orientation in Fig. 11.

Additionally, the experiment shows that the controller presents some robustness against the planar motion assumption. When the robots left the lane, there is a bump that makes the image to be crooked (see Fig. 12). The figure also serves as an example of the kind of images acquired by the robots in this experiment and the number of final matches computed at each iteration.

2) Experiment indoors: We have also tested the controller in an indoor environment. In this case the robots do not move forward and only turn $\left(v_{i}=0, \forall i\right)$. The results of the experiment are shown in Fig. 13. In this experiment we also plot the values of the control inputs of the three robots and the value of the epipoles. We observe that the epipoles and the control inputs present a larger variability than in simulations but nevertheless the robots end up in a configuration with all

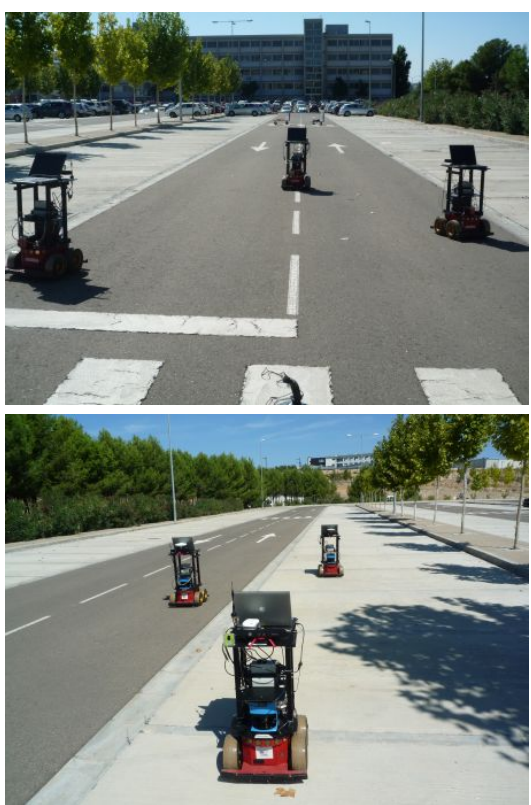

Fig. 11: Experiment with three robots outdoors. The initial configuration of the robots is shown on the top figure and the final configuration is shown on the bottom figure.

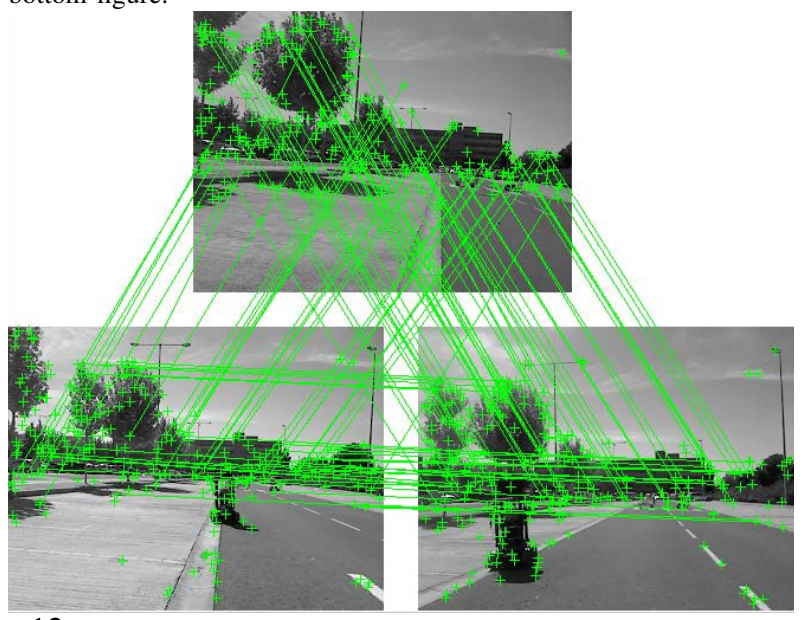

Fig. 12: Images acquired by the robots in the parking lot with the computed matches. The top image is slightly crooked due to the bump in the road (see the robot in the other two images). The controller seems to be robust to these small inclinations in the images.

the orientations aligned. ${ }^{1}$

3) Experiment using different cameras: Finally, as we have mentioned above, we have also evaluated the distributed control law equipping the robots with different cameras. In the last experiment two robots are still equipped with a Kinect sensor, but the third robot is equipped with a Unibrain camera. Figure 14 shows the initial and final configuration of the robots in this experiment. The unibrain camera has a larger value of $\alpha_{i}$ than the Kinect cameras, however, the value of $\beta$ has been set to 500 for the three robots. Looking at the positions of the robots we can see that the relative bearing is neither zero nor $\pi / 2$, which means that the final orientation should contain some error, as in eq. (25). However, the error in the final configuration is of the same magnitude as in the previous experiments, which means that in practice, the algorithm is more sensitive to the

\footnotetext{
${ }^{1}$ In the attached video there is another experiment where one robot is teleoperated to show convergence to consensus with a leader but due to space limitations no results are reported in the paper.
} 


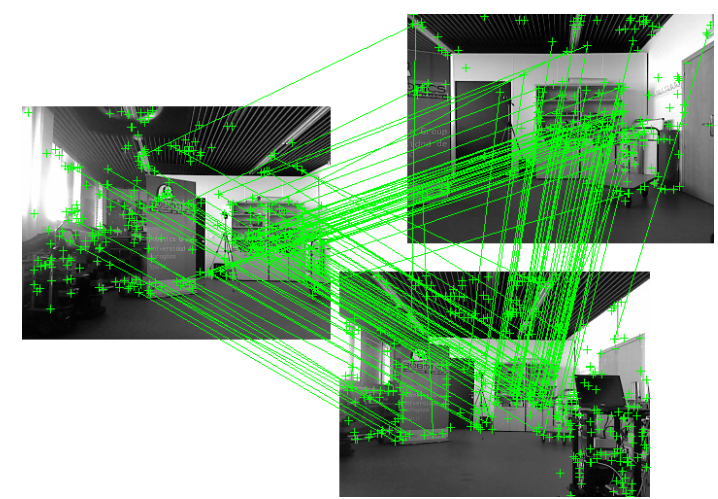

Matches between the robots

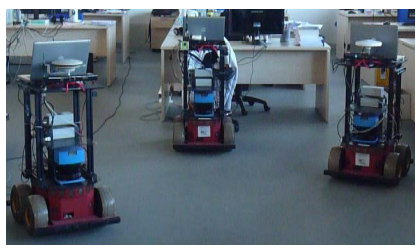

Initial configuration

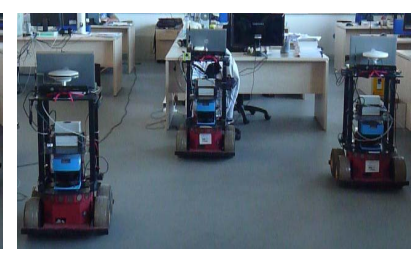

Final configuration
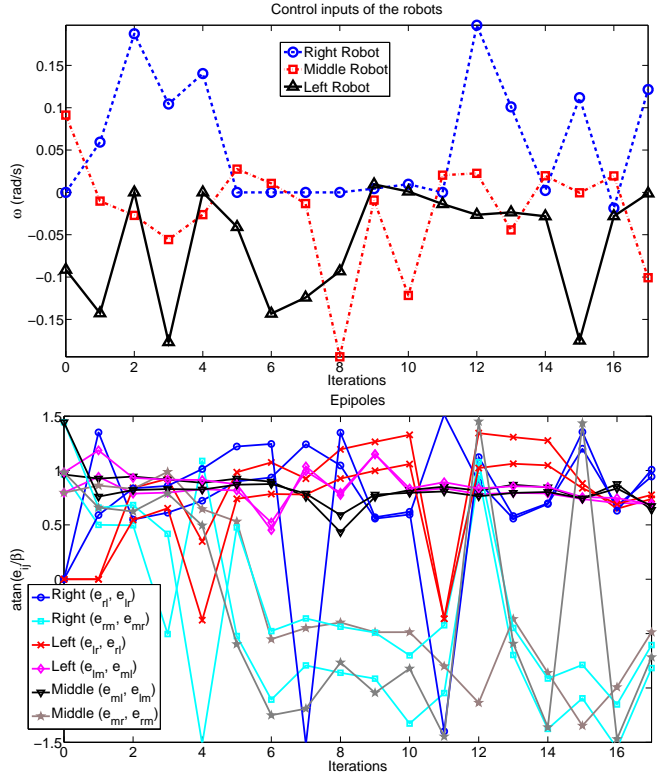

Fig. 13: Experiment in an indoors environment. In this case the robots have zero linear velocity, $v_{i}=0$, and only turn using the proposed controller. The two bottom graphics show the value of the control inputs and the epipoles respectively.

computation of the epipoles and the motion constraints of the robots than to the use of different cameras.

\section{CONCLUSIONS}

In this paper we have presented a new distributed controller that allows a team of robots to achieve a consensus in their orientations with monocular cameras. By using the epipoles computed between their images and the images of their neighbors, the robots reach a common orientation without topology or visibility limitations. Moreover, the controller is robust to the lack of some of the intrinsic parameters and can be used with just a coarse approximation of them, still reaching the consensus. In the paper we have also proved convergence to the consensus when the topology of the network changes over time, using this result to propose two

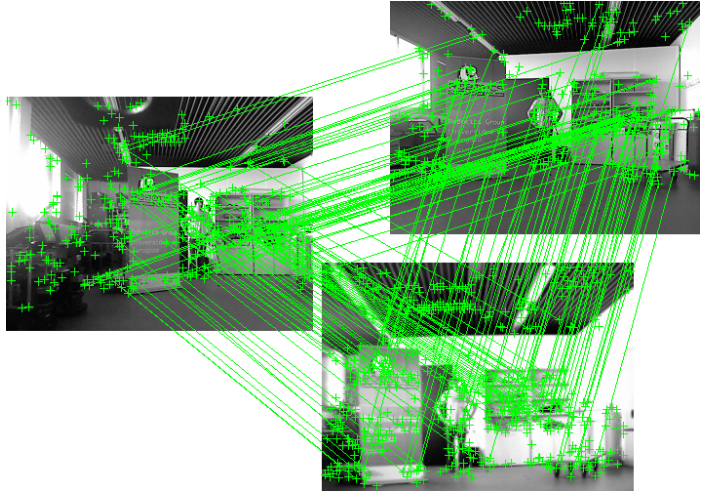

Matches between the robots

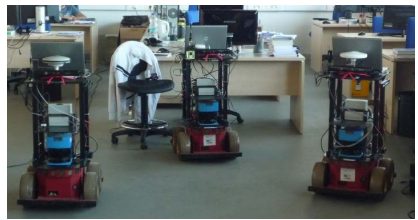

Initial configuration

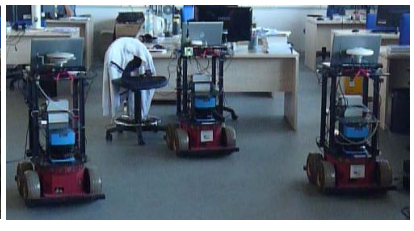

Final configuration
Fig. 14: In this experiment one of the robots is equipped with a different camera (Unibrain) than the other two (Kinect). On the top figure we show the matches between the three cameras. The bottom figures show the initial and the final configuration of the robots, in which the three robots are aligned despite the different calibrations.

distributed policies that reduce the number of fundamental matrices to be computed by the robots. Several simulations and experiments with real robots show the behavior of the approach in different situations. As for future perspectives, this work opens questions such as the use of acceleration techniques to reach the consensus in less time or if it is possible to use the proposed strategy also to automatically estimate the real intrinsic parameters of the camera.

\section{REFERENCES}

[1] W. Ren and R. W. Beard, Distributed Consensus in Multi-vehicle Cooperative Control, ser. Communications and Control Engineering. London: Springer-Verlag, 2008.

[2] F. Bullo, J. Cortés, and S. Martínez, Distributed Control of Robotic Networks, ser. Applied Mathematics Series. Princeton University Press, 2009, electronically available at http://coordinationbook.info.

[3] J. Cortes, "Global and robust formation-shape stabilization of relative sensing networks," Automatica, vol. 45, no. 12, pp. 2754 - 2762, December 2009.

[4] E. Montijano, and C. Sagues, "Distributed multi-camera visual mapping using topological maps of planar regions," in Pattern Recognition, vol. 44, no. 7, pp. 1528-1539, July 2011.

[5] A. Das, R. Fierro, V. Kumar, J. Ostrowski, J. Spletzer, and C. J. Taylor, "Vision based formation control of multiple robots," IEEE Transactions on Robotics and Automation, vol. 18, no. 5, pp. 813-825, October 2002.

[6] P. Urcola and L. Montano, "Cooperative robot team navigation strategies based on an environment model," in IEEE/RSJ International Conference on Intelligent Robots and Systems, October 2009, pp. 4577-4583.

[7] T. Ibuki, T. Hatanaka, M. Fujita, and M. W. Spong, "Visual feedback attitude synchronization in leader-follower type visibility structures," in 49th IEEE Conference on Decision and Control, December 2010, pp. 2486-2491.

[8] T. Gustavi and X. Hu, "Observer-based leader-following formation control using onboard sensor information," IEEE Transactions on Robotics, vol. 24, no. 6, pp. 1457-1462, December 2008.

[9] A. Jadbabaie, J. Lin, and A. S. Morse, "Coordination of groups of mobile autonomous agents using nearest neighbor rules," IEEE Transactions on Automatic Control, vol. 48, no. 6, pp. 988-1001, June 2003. 
[10] N. Mostagh and A. Jadbabaie, "Distributed geodesic control laws for flocking of nonholonomic agents," IEEE Transactions on Automatic Control, vol. 52, no. 4, pp. 681-686, April 2007.

[11] A. Sarlette and R. Sepulchre, "Consensus optimization on manifolds," SIAM Journal on Control Optimization, vol. 48, no. 1, pp. 56-76, January 2009.

[12] R. Sepulchre, "Consensus on nonlinear spaces," Annual Reviews in Control, vol. 35, no. 1, pp. 56-64, January 2011.

[13] J. Thunberg, E. Montijano, and X. Hu, "Distributed attitude synchronization control," in 50th IEEE Conference on Decision and Control and European Control Conference, December 2011, pp. 1962-1967.

[14] A. V. Savkin and H. Teimoori, "Decentralized navigation of groups of wheeled mobile robots with limited communication," IEEE Transactions on Robotics, vol. 26, no. 6, pp. 1099-1104, December 2010.

[15] K. D. Do, "Flocking for multiple elliptical agents with limited communication ranges," IEEE Transactions on Robotics, vol. 27, no. 5, pp. 931-942, October 2011.

[16] Y. Ma, J. Kosecka, and S. Sastry, "Vision guided navigation for a nonholonomic mobile robot," IEEE Transactions on robotics and automation, vol. 15, no. 3, pp. 521-536, June 1999.

[17] J. Courbon, Y. Mezouar, and P. Martinet, "Indoor navigation of a nonholonomic mobile robot using a visual memory," Autonomous Robots, vol. 25, no. 3, pp. 253-266, October 2008.

[18] A. Remazeilles and F. Chaumette, "Image-based robot navigation from an image memory," Robotics and Autonomous Systems, vol. 55, no. 4 pp. 345-356, April 2007.

[19] B. Gerkey, S. Thrun, and G. Gordon, "Visibility-based pursuit-evasion with limited field of view," The International Journal of Robotics Research, vol. 25, no. 4, pp. 299-315, April 2006.

[20] G. Lopez-Nicolas, M. Aranda, Y. Mezouar, and C. Sagues, "Visual control for multi-robot organized rendezvous," IEEE Transactions on Systems Man and Cybernetics: Part B, vol. 42, no. 4, pp. 1155-1168, April 2012.

[21] M. Schwager, B. Julian, M. Angermann, and D. Rus, "Eyes in the sky: decentralized control for the deployment of robotic camera networks," Proceedings of the IEEE, vol. 99, no. 9, pp. 1541-1561, September 2011.

[22] N. Mostagh, N. Michael, A. Jadbabaie, and K. Daniilidis, "Visionbased, distributed control laws for motion coordination of nonholonomic robots," IEEE Transactions on Robotics, vol. 25, no. 4, pp. 851-860, August 2009.

[23] R. Vidal, O. Shakernia, and S. Sastry, "Following the flock [formation control]," IEEE Robotics and Automation Magazine, vol. 11, no. 4, pp. 14-20, December 2004.

[24] R. Basri, E. Rivlin, and I. Shimshoni, "Visual homing: Surfing on the epipoles," International Journal of Computer Vision, vol. 33, no. 2, pp. 117-137, September 1999.

[25] G. Mariottini, G. Oriolo, and D. Prattichizzo, "Image-based visual servoing for nonholonomic mobile robots using epipolar geometry," IEEE Transactions on Robotics, vol. 23, no. 1, pp. 87-100, February 2007.

[26] G. López-Nicolás, C. Sagues, J. Guerrero, D. Kragic, and P. Jensfelt, "Switching visual control based on epipoles for mobile robots," Robotics and Autonomous Systems, vol. 56, no. 7, pp. 592-603, July 2008.

[27] G. Erinc and S. Carpin, "Image-based mapping and navigation with heterogeneous robots," in IEEE/RSJ International Conference on Intelligent Robots and Systems, October 2009, pp. 5807-5814.

[28] E. Montijano, J. Thunberg, X. Hu, and C. Sagues, "Multi-robot distributed visual consensus using epipoles," in 50th IEEE Conference on Decision and Control and European Control Conference, December 2011, pp. 2750-2655.

[29] — - "Distributed policies for neighbor selection in multi-robot visual consensus," in 2nd Workshop on Visual Control of Mobile Robots (ViCoMoR) at IEEE/RSJ International Conference on Intelligent Robots and Systems, October 2012, pp. 13-18.

[30] Y. Ma, S. Soatto, J. Kosecka, and S. S. Sastry, An Invitation to 3D Vision. SpringerVerlag, 2004.

[31] J. Marsden and A. Weinstein, Calculus, Volume III, Chapter 16, ser. Applied Mathematics Series. Springer, 1985.

[32] A. Bacciotti and L. Mazzi, "An invariance principle for nonlinear switched systems," System and Control Letters, vol. 54, no. 11, pp. 1109-1119, November 2005.

[33] D. Cheng, J. Wang, , and X. Hu, "An extension of LaSalle's invariance principle and Its application to multi-agent consensus," IEEE Transactions on Automatic Control, vol. 53, no. 7, pp. 1765-1770, July 2008.
[34] J. Wang and D. Cheng, "Extensions of LaSalles invariance principle for switched nonlinear systems," in Proceedings of the 17th World Congress The International Federation of Automatic Control, Seoul, Korea, July 2008, pp. 14397-14402.

[35] D. Lowe, "Distinctive image features from scale-invariant keypoints," International Journal of Computer Vision, vol. 60, no. 2, pp. 91-110, November 2004.

[36] R. Hartley and A. Zisserman, Multiple View Geometry in Computer Vision. Cambridge: Cambridge University Press, 2000

[37] D. Tardioli, A. Mosteo, L. Riazuelo, J. Villarroel, and L. Montano, "Enforcing network connectivity in robot team missions," The International Journal of Robotics Research, vol. 29, no. 4, pp. 460-480, April 2010.

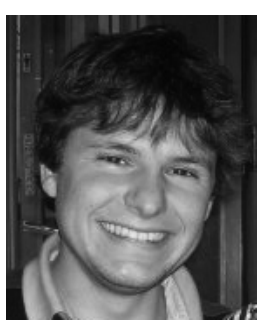

Eduardo Montijano (M'12) received the M.Sc. and Ph.D. degrees from the Universidad de Zaragoza, Spain, in 2008 and 2012 respectively, supervised by Prof. Carlos Sagüés. He is currently a Professor at Centro Universitario de la Defensa, in Zaragoza, Spain. His research interests are computer vision and consensus algorithms applied to multiple robots.

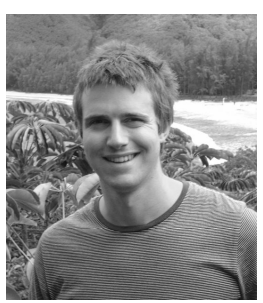

Johan Thunberg was born in Stockholm, in 1982. $\mathrm{He}$ has received a Master of Science degree in Engineering Physics from the Royal Institute of Technology (KTH) in Stockholm, Sweden. After finishing his Master he has worked as a research assistant at the Swedish Defence Research agency (FOI), and at ENEA, a swedish software company specialized in embedded systems.

Currently he is a graduate student in applied mathematics at the Royal Institute of Technology (KTH) in Stockholm, and is funded by the Swedish National Space Technology Research Programme (NRFP) and the Swedish Research Council (VR). His supervisor is Professor Xiaoming Hu.

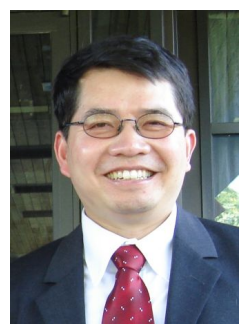

Xiaoming Hu received his B.S. degree from the University of Science and Technology of China in 1983, and his M.S. and Ph.D. degrees from Arizona State University in 1986 and 1989 respectively He served as a research assistant at the Institute of Automation, the Chinese Academy of Sciences, from 1983 to 1984. From 1989 to 1990 he was a Gustafsson Postdoctoral Fellow at the Royal Institute of Technology, Stockholm, where he is currently a professor of Optimization and Systems Theory. His main research interests are in nonlinear control systems, nonlinear observer design, sensing and active perception, motion planning, control of multi-agent systems, and mobile manipulation.

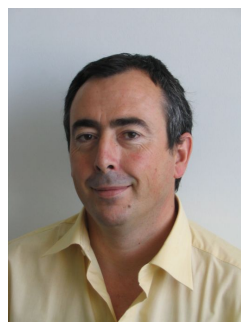

Carlos Sagïés (M'00, SM'11) received the M.Sc. and Ph.D. degrees from the Universidad de Zaragoza, Spain. During the course of his Ph.D. he worked on force and infrared sensors for robots. Since 1994 he has been Associate Professor and, since 2009 Full Professor with the Departamento de Informática e Ingenieía de Sistemas, Universidad de Zaragoza, where he has also been Head Teacher. His current research interest includes control systems, computer vision, visual robot navigation and multivehicle cooperative control. 